\title{
Symposium der Alkenpreisträger in Bussum/Amsterdam Niederlande, Dezember 1989
}

\section{Neurourologie und Urodynamik}

Das wissenschaftliche Treffen der Alkenpreisträger eröffnete L. Hertle, Universität Bochum, mit seinem Vortrag

\section{„Neurotransmission und Signalübertragung an der glatten Muskulatur des menschlichen Harntraktes“}

\section{Koautor dieser prämierten Arbeit war H. Nawrath}

Die biologische Bedeutung und der Wirkmechanismus zahlreicher Neurotransmitter an der glatten Muskulatur des menschlichen Harntraktes sind weitgehend unbekannt. Kontraktion und Relaxation der glatten Muskelzellen sind im wesentlichen abhängig von der freien intrazellulären Kalziumionenkonzentration. Die Interaktion eines Agonisten mit seinem spezifischen Rezeptor an der Zelloberfläche führt, über zum Teil unbekannte intrazelluläre Signalwege, zu einer Beeinflussung der freien intrazellulären Kalziumkonzentration. Am Beispiel des Noradrenalins (des Uberträgerstoffes des sympathischen Nervensystems) wurde gezeigt, daß die Wirkung von Neurotransmittern an unterschiedlichen Geweben des Urogenitaltraktes sehr verschieden ist. So induziert Noradrenalin an Nierenbecken und -kelchen konzentrationsabhängig lang anhaltende Dauerkontraktionen, während der gleiche Neurotransmitter am normalen Harnleiter kaum Effekte hervorruft oder allenfalls kurzdauernde phasische Kontraktionen induziert. Demgegenüber induziert Noradrenalin an Streifenpräparaten aus den dilatierten Anteilen von Megaureteren kräftige tonische Kontraktionen. Substanzen mit einem modulatorischen Effekt an Kalziumionenkanälen (sogenannte Kalziumantagonisten oder Kalziumagonisten) haben nur geringe Wirkungen auf die von Noradrenalin induzierten tonischen Kontraktionen. Die genannten Befunde verweisen auf ausgeprägte ultrastrukturelle Unterschiede zwischen den glattmuskulären Systemen im Bereich des ableitenden Harntraktes. Offensichtlich unterscheiden sich glatte Muskelzellen in Abhängigkeit von ihrer topographischen Herkunft wesentlich im Hinblick auf ihre Kalziumaktivierungssysteme. Die Wirkung von Noradrenalin wird wahrscheinlich über einen im Bereich des Harntraktes bisher nicht bekannten intrazellulären Signalweg vermittelt, der möglicherweise über die Inositpolyphosphate abläuft. Die weitere Klärung der zellulären Wirkungen von Neurotransmittern an glatten Muskelzellen des Harntraktes ist von entscheidender Bedeutung für das Verständnis normaler und pathologischer glattmuskulärer Aktivität.

Akt. Urol. 21 (1990) 290-298

(C) Georg Thieme Verlag Stuttgart · New York

\section{„Diagnostische Miktions-Parameter der Evakuations-Phase: Harnblasenkontraktilität und Harnröhrenwiderstand"}

(R. Mastrigt, Urologische Universitätsklinik, Erasmus Universität Rotterdam)

Während der Evakuationsphase kann die Harnblase durch mehrere Parameter charakterisiert werden. Unter physiologischen Bedingungen genügen zur angemessenen Charakterisierung des Systems Kenntnisse über die Blasenkapazität und den Restharn nach Miktion. Diese beiden Größen können deshalb physiologische Parameter genannt werden. Bei Dysfunktion des Systems sind jedoch zur akkuraten und vollständigen Beschreibung des Grades der Dysfunktion zusätzliche Parameter erforderlich. Sie können symptomatische Parameter genannt werden. Die maximale Harnflowrate $(\mathrm{Qmax})$ stellt einen solchen Parameter dar. Die Behandlung der Dysfunktion erfordert Kenntnisse über deren Ursache. Wenn diese Kenntnisse in objektive Größen zusammengefaßt werden können, werden sie zu diagnostischen Parametern.

Während der Entleerungsphase arbeitet die Harnblase vergleichbar einer Pumpe, die Druck zum Harntransport durch die Urethra erzeugt. Voraussetzung hierfür ist die Kontraktionsfähigkeit der Harnblase, die charakterisiert ist durch das Verhältnis zwischen Druck in der Harnblase und der Geschwindigkeit, mit der sich die Blasenwand kontrahiert. Dieses Verhältnis reflektiert das basale KraftGeschwindigkeitsverhältnis des sich kontrahierenden Blasenmuskels. Wesentliche Eigenschaft der Harnröhre unter der Miktion ist ihr Flowwiderstand, worunter der zu einer gewissen Flowrate erforderliche Druck verstanden wird. Beide wesentliche Eigenschaften der Blase und Urethra können in Form des Verhältnisses zwischen Detrusordruck und Flowrate beschrieben werden. Durch Projektion beider Verhältnisse gegeneinander kann der aktuelle Druck und die Flowrate, mit welcher die Miktion stattfindet, bestimmt werden (Abb. 1).

Urodynamische Untersuchungen erfolgen mit dem Ziel, Informationen über den Zustand des Harntraktes zu erhalten, Dysfunktionen zu entdecken und gegebenenfalls zu behandeln. Ein häufig angewandtes Verfahren ist die Zystometrie: Detrusordruck und Flowrate werden als Funktion der Miktionszeit bestimmt. Maximale Flowrate und Druck gemessen zu bestimmten Zeitpunkten werden gewöhnlich als Parameter verwandt. Diese Parameter repräsentieren jedoch nicht die wesentlichen Eigenschaften des Systems, nämlich die Blasenkontraktilität und den Urethrawiderstand. Sie sind deshalb keine diagnostische Parameter, bestenfalls symptomatische. Diagnose und Behandlung können nicht ausgehend von diesen Parametern bestimmt werden. 


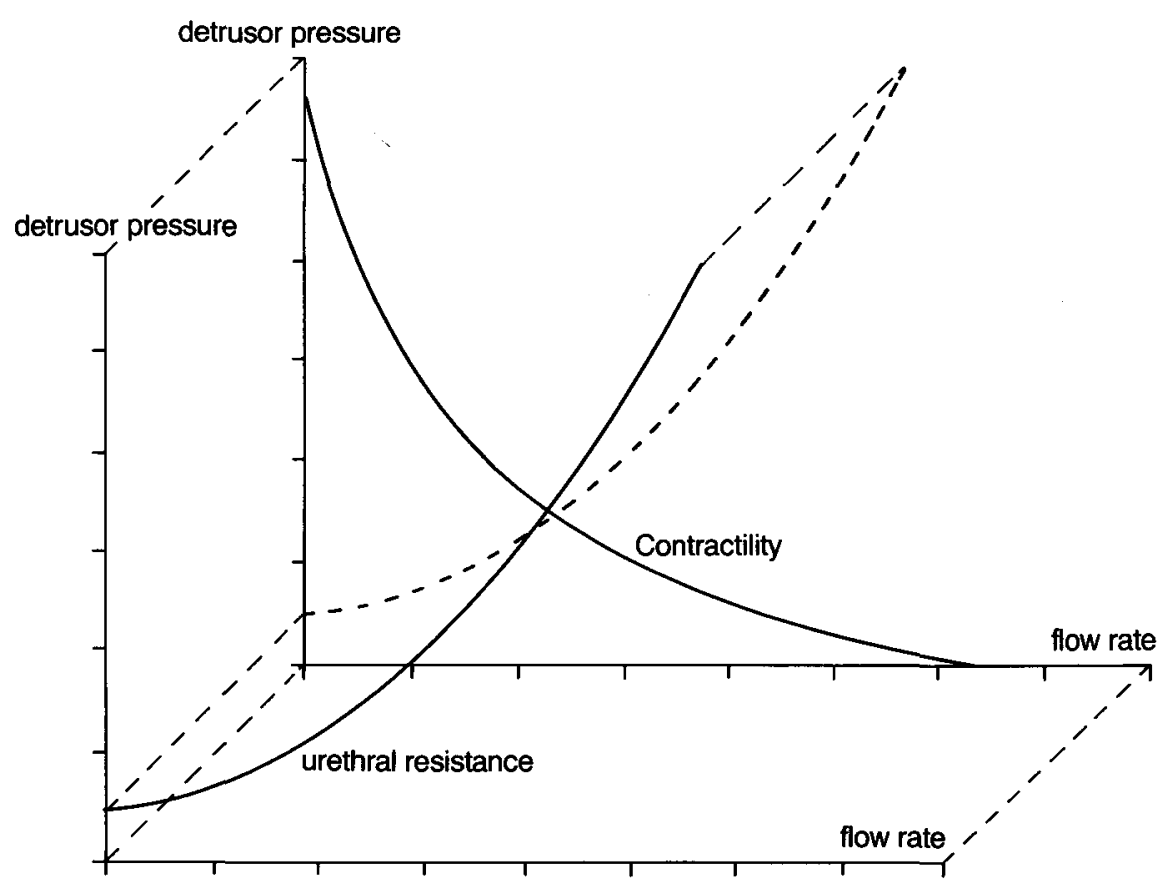

Abb. 1 Durch Projektion des Detrusordruckes und Flowrate gegeneinander können der aktuelle Druck und die Flowrate, mit welcher Miktion stattfindet, bestimmt werden.

Es wurde ein Computerprogramm entwikkelt, das den Anschluß der Urodynamischen Apparatur an einen I.B.M. compatibelen P.C. ermöglicht. Das Programm (CLIM) speichert Detrusordruck- und -Flowrate-Signale und erlaubt die Kalkulation diagnostischer Parameter. Es können zwei Parameter bestimmt werden, die unabhängig voneinander den Urethrawiderstand quantifizieren. Ein Parameter wird abgeleitet vom isometrischen Druckanstieg, der der Miktion vorangeht (U/1), der zweite Parameter gibt den errechneten Schnittpunkt der Druck-Flowrate-Kurve mit der Druckachse wieder (URA). Beide Parameter sind bei obstruktiven Patienten erhöht und normalisieren sich nach Aufhebung der Obstruktion (Mastrigt et al., Neurourol. Urodyn. 7:226-228, 1988). Maximale Kontraktions-Geschwindigkeit (V max) und maximaler isometrischer Druck (PO) charakterisieren die Kontraktilität der Harnblase. Diese Kontraktionsparameter können in jeder Druck-Flow-Untersuchung bestimmt werden. Sie repräsentieren verschiedene Aspekte der Kontraktilität. Bei Patienten mit Obstruktion steigt PO signifikant an, während V max unbeeinflußt bleibt. Ein Parameter, der beide Aspekte der Kontraktilität in Form einer approximierten Leistung bezogen auf die Blasenwandoberfläche kombiniert, kann ebenfalls abgeleitet werden. Dieser Parameter W max kann zur Voraussage der Miktionsfähigkeit nach TUR verwandt werden. Wird ein geringer W max-Wert präoperativ bestimmt, ist mit einem erhöhten Restharn nach TUR zu rechnen. Für die Harnblase sind daher die Parameter PO und V max oder alternativ W max und für die Urethra die Parameter URA und U/1 als diagnostische Parameter anzusehen.

\section{R. Hohenfellner sprach über}

„Neuropathische Dysfunktion der Harnblase: Therapeutische Aspekte“

\section{(Urologische Universitätsklinik Mainz)}

Über einen Zeitraum von 25 Jahren wurden nahezu 300 Kinder mit Meningomyelozelen multidisziplinär behandelt und verfolgt. Anhand einer retrospektiven Analyse wurden ermittelt: (1) prognostische Faktoren mit Voraussagewert für mögliche Verschlechterung des oberen Harntraktes und für Erreichen der Harnkontinenz, (2) optimale Intervalle für Kontrolluntersuchungen bei hoch und weniger gefährdeten Kindern, (3) Ergebnisse der konservativen und medikamentösen Therapie, des intermittierenden Katheterismus und des Blasentrainings und (4) die Ergebnisse der operativen Behandlung (Blasenaugmentation, kontinente Harnableitung und Sphinkterimplantation).

Ziel der therapeutischen Bemühungen bei MMC-Kindern ist die Erhaltung einer normalen Nierenfunktion und Erreichen der Kontinenz. Der Erhaltung der Nierenfunktion haben sich Bemühungen zum Erreichen der Kontinenz unterzuordnen. Die Nierenfunktion wird bedroht durch erhöhten BlasenauslaBwiderstand und ungehemmte Blasenkontraktionen (hyperreflektive Blase). Beide gilt es zu beseitigen durch Blasenhalsrekonstruktion, gegebenenfalls in Kombination mit einer Blasenaugmentationsplastik bei hyperreflektiven kleinen Blasen $(<100 \mathrm{ml})$. Bei geringem Blasenauslaßwiderstand $(<15 \mathrm{~cm})$ mit Inkontinenz, ist Kontinenz sowohl durch Faszienzügelplastik als auch durch Implantation einer AMS-Sphinkterprothese zu erreichen.

Der Zustand des oberen Harntraktes (Dilatation) nach den geschilderten Maßnahmen bzw. bei konservativen Vorgehen (Oxybutinin bei hyperreflektiver Bla- 
se) wird echographisch im 3-Monate-Rhythmus evaluiert, um bei Problemen rechtzeitig Zusatzmaßnahmen einleiten zu können. $\mathrm{Zu}$ diesen zählen: intermittierender Katheterismus, Antirefluxoperation, Schaffung eines Niederdrucksystems (Colon-Konduit) oder einer kontinenten Harnableitung. Credé-Manöver zur Förderung der vollständigen Blasenentleerung, eine abwartende Haltung bei Dilatation des oberen Harntraktes oder operative Korrektion der Inkontinenz bei Stauung der oberen Harnwege, sind fehlerhafte Entscheidungen und deshalb in den geschilderten Situationen kontraindiziert.

Der Wandel in den Harnableitungsformen spiegelt sich bei Kindern mit neurogenen Blasen im eigenen Krankenmaterial und im Schrifttum der letzten Dekade wieder: vom Ileumkonduit mit seiner hohen Komplikationsrate ( $80 \%$ ) auf lange Sicht, zum Kolonkonduit mit antirefluxiver Ureterimplantation zur kontinenten Niederdruckharnableitung mit detubularisierten Darmsegmenten. Harnableitung kann unumgänglich sein bei Kindern mit ernsthaften orthopädischen Schäden (Kyphoskoliose) oder Adipositas, die intermittierenden Katheterismus nicht zulassen, wegen fehlender geistiger und körperlicher Reife, oder bei Ablehnung des Katheterismus selbst. Der intermittierende Katheterismus ist nicht frei von Komplikationen: Infektionen, $\mathrm{Ma}$ krohämaturie, Fausse Route mit Abszessen der Prostata, Perforation der augmentierten Blase. Noch schwer abschätzbar ist das Risiko der Entstehung/Förderung eines malignen Tumors in der augmentierten Blase (oder in der kontinenten Harnableitung).

So fließen in den Entscheidungsprozeß zum richtigen Vorgehen viele Überlegungen ein. Das ideale Verfahren besteht nicht. Jedes Verfahren muß bei diesen Kindern, denen die Voraussetzungen $\mathrm{zu}$ einem weitgehend normalen Leben mit normaler Lebenserwartung angeboten werden sollen, auf seinen Langzeitwert geprüft und mit kritischer Distanz angewandt werden.

\section{Uro-Onkologie}

\section{H. J. Schamhart sprach über}

\section{„Interleukin 2 und Interleukin 6 im Harn: BCG-Behandlung oberflächlicher Blasenkarzinome ${ }^{\text {* }}$}

Koautoren waren R. Vleeming, T.M. de Reijke und $K$. H. Kurth, Urologische Universitätsklinik, Universität von Amsterdam

BCG hat sich als wirksame Substanz zur Behandlung (Cis der Harnblase) und adjuvanten Immuntherapie von Blasenkarzinomen der Kategorie $\mathrm{Ta} / \mathrm{T} 1$ erwiesen. Der Wirkmechanismus der Substanz ist jedoch noch nicht vollständig geklärt. Unter BCG-Behandlung wurden lokale Entzündungsreaktionen, eine erhöhte Aktivität von Subpopulationen der T-Zellen und eine erhöhte Ausscheidung von Zytokinen beobachtet und als wichtig für den Therapieerfolg gewertet. In der vorgestellten Untersuchung wurde die $\mathrm{Ki}$ netik der Zytokine Interleukin 2 (IL-2) und IL-6 als Indikator der Proliferation von T-Zellen und Makrophagen unter
BCG-Therapie im Harn bestimmt. Patienten mit oberflächlichen Blasentumoren wurde BCG-Pasteur $150 \mathrm{ml}$ wöchentlich über einen Zeitraum von sechs Wochen instilliert. Über einen Zeitraum von 24 Stunden wurden Harnprobe und „Bladder wash-outs“ vor und nach BCG-Instillation versammelt. IL-2 wurde mit einem Radioimmunassay bestimmt. Zur Bestimmung der IL-6 wurde ein Bioassay verwandt (BZell-Hybridoma-Zellinie). Die mit der Irrigation (Bladder wash-out) erhaltenen Zellen wurden gezählt (FACS) und Zytospins hergestellt. Zur Bestimmung der T3-Lymphozytenpopulation wurden immunhistochemische Verfahren angewandt. Ein Anstieg der IL-6 wurde bereits in der zweiten bis dritten Behandlungswoche beobachtet. Dieser Anstieg ging einher mit einer Zunahme von Zellen, die aus dem Blasenirrigat drei Stunden nach BCG-Instillation gewonnen wurden. Diese Zunahme betraf vornehmlich neutrophile Granulozyten. IL-2 stieg ab der vierten bis fünften Behandlungswoche meßbar an und ging stets einher mit einem signifikanten Anstieg der T-Lymphozyten. Obwohl in der gesamten Patientenpopulation der Anstieg der Zytokine nicht einheitlich erfolgte, wurden Peak-Werte von $25 \mathrm{fmol} /$ mol Kreatinin für IL-6 und für IL-2 von $250 \mathrm{fmol} / \mathrm{mol} \mathrm{Kreati-}$ nin gemessen.

Die Reaktion der tumorbefallenen Harnblase auf BCG kann deshalb in zwei aufeinanderfolgende Prozesse unterteilt werden: zunächst erfolgt eine entzündliche Reaktion ablesbar am Anstieg der IL-6 und vornehmlich der neutrophilen Granulozyten, hierauf folgt ein Anstieg der IL2 und der T-Lymphozyten. Letzteres läßt auf eine T-Zell gesteuerte immunologische Reaktion schließen, die entweder gegen $B C G$ und/oder Tumorantigen gerichtet ist. Es haben sich Hinweise ergeben, daß dem Anstieg der Zytokinen unter Immuntherapie prognostische Bedeutung zukommt.

Den gleichen Themenkreis behandelte $P$. A. Steerenberg, Rijks Instituut voor Volksgezondheid, Bilthoven (NL) in seinem Vortrag

\section{„Immunologische und histologische Aspekte der BCG-Immuntherapie beim oberflächlichen Harnblasenkarzinom"}

Koautoren dieser Untersuchung waren A. B. M. van der Meijden, J. E. Ruitenberg und W. H. de Jong

Harnproben wurden von Patienten während einer sechs Wochen dauernden Behandlung mit BCG-RIVM versammelt. IL-2 wurde in dialysierten Harnproben mittels eines In-vitro-Bioassays bestimmt, die Inkorporation von markiertem Thymidin in Zellen der T-Zell-linie CLL-16 galt als Indikator für die Anwesenheit von IL-2. In Harnproben, gewonnen vor BCG-Instillation und $24 \mathrm{~h}$ nach Instillation, fand sich kein IL-2, hingegen konnte IL-2 in den ersten Stunden nach BCG-Verabreichung nachgewiesen werden. Hieraus kann gefolgert werden, daß die IL-2-Kinetik im direkten Zusammenhang mit der BCG-Verabreichung steht. IL-2-Werte stiegen nach der dritten BCG-Instillation an. Bei einigen Patienten fand sich dieser IL-2-Anstieg nicht, auch nicht nach der 22. Instillation. Inwieweit der fehlende IL-2Anstieg prognostisch bedeutsam ist, wird weiter untersucht. In die Blase verabreichtes BCG wird zum größten Teil innerhalb von 24 Stunden mit dem Harn wieder ausgeschie- 
den. Vermutlich kommt nur ein kleiner Anteil des BCG in direkten Kontakt mit der Harnblase und leitet dort immunologische bzw. entzündliche Prozesse ein. BCG in die intakte Blase von Zwergschweinen instilliert, führt zur Induktion von Granulomen der Harnblase und der juxtaregionalen Lymphknoten, ohne das Blasenepithel direkt zu schädigen. Verschiedentlich wurde die Frage aufgeworfen, ob die Mykobakterien am Urothel festsitzen oder in das Epithel penetrieren müssen, um die entzündliche Reaktion herbeizuführen. Zur Beantwortung dieser Fragen wurden Rasterund Elektronenmikroskopie der Blase von Zwergschweinen nach BCG Instillation durchgeführt. Bei den meisten Tieren konnten keine adhährente Mykobakterien am Blasenepithel nachgewiesen werden, selbst nicht wenn diese Blasen vorab durch Elektrokauter geschädigt wurden. In den wenigen Fällen, in denen sich adhärente Mykobakterien auf dem Blasenepithel fanden, erfolgte keine Phagozytose in die Epithelzellen. Es ist fraglich, ob dieser Befund der normalen Blase auch für die Tumorblase gilt, da bei Verwendung der Blasentumorlinie T-24 sehr wohl Phagozytose von BCGMykoorganismen in die Tumorzelle stattfand.

K. H. Kurth, Urologische Universitätsklinik, Universität Amsterdam, berichtete über

\section{„Häufigkeit von Progression und Überleben bei Patienten mit Blasentumoren der Kategorie Ta/T1“}

Koautoren waren Mitglieder der EORTC-Genito-Urinary Group

425 Patienten mit Blasentumoren der Kategorie Ta/T1 wurden im Mittel viereinhalb Jahre verfolgt (Zeitraum 1 bis 9 Jahre). In dieser prospektiven Untersuchung wurden die Patienten randomisiert behandelt mit alleiniger transurethraler Resektion oder mit Resektion gefolgt von adjuvanter Chemotherapie. Während die adjuvante Chemotherapie signifikant das Intervall bis zum ersten Rezidiv verlängerte und ebenfalls signifikant die Häufigkeit von Operationen wegen Tumorrezidiv verminderte, hatte die adjuvante Chemotherapie keinen Einfluß auf sowohl Tumorprogression $(\geq \mathrm{T} 2)$ als auch Überleben. Tumorprogression wurde bei 54/392 Patienten beobachtet (13,7\%). Die Häufigkeit von Tumorprogression unterschied sich im Behandlungsarm mit alleiniger transurethraler Resektion nicht von der in den Behandlungsarmen mit adjuvanter Chemotherapie. Andererseits war die Tumorprogression deutlich abhängig von der behandelten T-Kategorie. Während lediglich $7 \%$ der Patienten mit Ta-Blasentumoren Tumorprogression zeigten ( $\geq \mathrm{T} 2)$ betrug sie für T1-Blasentumoren nahezu $25 \%$. Neben der T-Kategorie war die Tumorprogression deutlich dem Malignitätsgrad korreliert. Verglichen mit G1-Tumoren wurde Tumorprogression viermal häufiger bei G2- und sechsmal häufiger bei G3-Tumoren beobachtet. 115/420 Patienten verstarben im Followup, 38/115 Patienten an einer malignen Erkrankung. Da während der Verlaufsbeobachtung bei 46 Patienten ein Zweittumor außerhalb des Harntraktes diagnostiziert wurde, konnte nicht mit Sicherheit festgestellt werden, ob die 38 verstorbenen Patienten (9\%) am Blasenkarzinom oder an der zweiten malignen Grunderkrankung verstarben. Die Analyse der Überlebungskurven in den verschiedenen Behandlungsarmen zeigte keinen Vorteil für adjuvante Chemotherapie im Sinne einer Lebensverlängerung, gleichgültig ob diese Analyse mit Einschluß aller 115 verstorbenen Patienten oder lediglich mit Berücksichtigung der 38 am malignen Tumor Verstorbenen erfolgte. Die Schlußfolgerung aus dieser Langzeitanalyse ist, daß einerseits signifikant die Rezidivrate/ Jahr durch adjuvante Chemotherapie günstig beeinflußt wird $(p<.000)$, andererseits adjuvante Chemotherapie mit den verwandten Behandlungsschemata nicht zu einer nachweisbaren Verminderung der Tumorprogression oder zu einer Lebensverlängerung führt. Entscheidender als adjuvante Maßnahmen bestimmen prognostische Faktoren wie T-Kategorie, Malignitätsgrad und, in geringeren Umfang, die frühere Rezidivrate das weitere Schicksal der Patienten.

H. J. Tanke, Abteilung für Zytochemie und Zytometrie, Universität Leiden, sprach über

\section{„Zukünftige Rolle der Nukleinsäuren-Analyse in der urologischen Onkologie durch In-situ-Hybridisierung und Bildanalyse“}

Entwicklungen in der Molekularbiologie und der Immunologie, die Verfügbarkeit von monoklonalen Antikörpern und Nukleinsäure-Probes in Kombination mit der Ausbreitung der Mikroelektronik und der Computertechnik, führten zu einem Durchbruch in der Krebsforschung auf genetischem Niveau. Methoden zur Untersuchung des menschlichen Genoms auf dem Niveau des einzelnen Gens haben ihren Wert sowohl in der klinischen Genetik als auch in der Onkologie und der pränatalen Diagnostik gezeigt. Bei dieser Untersuchung bedient man sich radioaktiver Markersubstanzen oder alternativ fluoreszierender Stoffe. Die gegenwärtige Forschung ist darauf gerichtet, nicht-radioaktive Methoden zum mikroskopischen Nachweis und zum Quantifizieren von multiplen Makromolekülen (Proteinen, Nukleinsäuren) sowohl im Gewebe als auch in individuellen Zellen zu entwickeln. Hiervon wird eine größere Sensitivität erwartet als von jener erreicht mit radioaktiven Methoden oder mit dem Fluoreszenz-Assay. Die Entwicklung auf dem Lasergebiet und von Kameras mit Bildverstärkermöglichkeit oder von neuartigen Detektoren (charge-coupled device-CCD) erlaubt im Prinzip den Nachweis eines einzigen fluoreszierenden Moleküls. Videoverstärkte Lichtmikroskopie und die digitale Verarbeitung der Fluoreszenzsignale haben die Möglichkeit des Einsatzes dieser Systeme in der Zellbiologie erweitert. Die theoretisch erwartete Sensitivität wird mit diesen Systemen jedoch kaum erreicht. Ursache hiervon ist die Autofluoreszenz biologischen Materials und die Fluoreszenz der optischen Komponenten des Fluorometers. Die Autofluoreszenz kann selektiv durch die Verwendung von Material mit verzögerter Lumineszenz und relativ langsamen Verfallscharakteristiken unterdrückt werden. Dies trifft auf die Elemente der seltenen Erden zu. Da die störende Autofluoreszenz meist Folge schnellen Verfalls ist, können durch Anwendung von zeitaufgelöster Fluorometrie die Fluoreszenz mit Halbwertszeiten in der Größenordnung von Nanosekunden, die langsame Lumineszenz als Folge der Markierung und die schnelle Autofluoreszenz voneinander getrennt werden. Natürliche zelluläre Makromoleküle mit einer langsamen Lumineszenz sind relativ selten. In den vergangenen Jahren wurde in Leiden eine sich nicht erschöpfende Lumineszenzmarkierung entwickelt, mit der 
Möglichkeit, die oben geschilderten Probleme zu umgehen. Die Markierung zeigt eine relativ stark verzögerte Lumineszenz mit einer Halbwertszeit von mehreren hundert Mikrosekunden. Sie wird erreicht mit anorganischem Phosphor (Yttrium-Oxysulfïdkristalle aktiviert mit Europium) mit einer Teilchengröße von 50 bis $500 \mathrm{~nm}$. Die Herstellung erfolgt aus lumineszierendem Material, wie es in Fensehgeräten verwandt wird. Makromoleküle wie Immunglobuline, Avidin oder Protein A, werden an der aktivierten Oberfläche der Kristalle gebunden und behalten ihre immunologischen Eigenschaften. Die immunzytochemische Lokalisation von Antigenen auf Zellmembranen konnte unter Verwendung von Phosphorgranulaten demonstriert werden.

Der selektive Nachweis von mit verzögert lumineszierendem Immunphosphor markierten Antigenen wurde demonstriert durch die effektive Unterdrückung von schnell abnehmender Autofluoreszenz biologischen Materials mittels eines, für diesen Zweck speziell konstruierten, zeitauflösenden Fluoreszensmikroskops. Die Entwicklung einer großen Gruppe lumineszierender Stoffe mit relativ geringem Molekulargewicht und die hierdurch erreichbaren guten zytochemischen Penetrations-Eigenschaften, ist eine Herausforderung an die synthetische Chemie. Die Fluoreszenz ist ein Phänomen, das einhergeht mit schwachen Lichtsignalen, deshalb müssen, um die Sensitivität der Methode weiter zu verbessern, wirksam arbeitende Detektoren eingesetzt werden. Hierbei sind zwei Methoden zu erwähnen: 1) die Verwendung der Videotechnik für Videoverstärkte-Fluoreszenzmikroskopie (VIFM) und die Videoverstärkte-Lichtmikroskopie (VELM) und 2) die Verwendung von speziellen Kameras (CCD).

Die CCD-Sensoren bestehen aus einem Register, gebildet aus dicht aufgereihten Metall-Oxyd-Silikon(MOS)-Sensoren, die speichern und in der Folge Elektronen transferieren, die nach Lichteinfall eingeleitet werden. Die Struktur des Systems gewährleistet geometrische Zuverlässigkeit. Da die Anzahl der Elektronen direkt korreliert ist mit der Häufigkeit der anfallenden Photonen, arbeitet das Gerät linear über einen großen Meßbereich. Darüber hinaus zeigen CCD-Kameras nur ein geringes Hintergrundsgeräusch, wenn sie thermoelektrisch oder kryogenetisch gekühlt werden.

Eine wichtige Anwendung der neuen Techniken ist der Nachweis kleinster Mengen eines Makromoleküls wie kleinste DNA-Sequenzen oder spezifische Messenger-RNA nach zytochemischer Färbung mit spezifischen nicht-radioaktiven Nukleinsäure-Probes (Detektionsgrenze ca. $1 \mathrm{~KB}$ ). Die Entwicklung von nichtradioaktiver In-situHybridisierung und die Immunozytochemie erlauben prinzipiell Untersuchungen an der morphologisch intakten individuellen Zelle auf Molekülebene. Hiermit eröffnen sich vielversprechende diagnostische Möglichkeiten, besonders auf dem Gebiet der Genetik und Onkologie. Für die urologische Onkologie im allgemeinen und für die Diagnostik im beson- deren finden die obengeschilderten Methoden Eingang in laufende Untersuchungsprojekte. Mit Hilfe der Molekularbiologie kann nachgewiesen werden, daß die Amplifikation von Onkogenen häufig eine wichtige Rolle bei malignen Prozessen der Blase und der Prostata spielt. Läßt sich eine solche Amplifikation auf Zellniveau nachweisen, ist die Frühdiagnose von Tumoren vermutlich zu verbessern (z. B. an zytologischem Material der Harnblase). Die zweite Möglichkeit ist die Verwendung chromosomenspezifischer Probes, mit denen numerische Chromosomenabweichungen (und in der Zukunft eventuell auch Translokationen) während der Interphase von Zellen nachgewiesen werden können. Relativ einfach können hiermit Markerchromosome aufgespürt werden und ist es möglich diploide Tumoren, bei denen eine bessere Prognose unterstellt wird, aufgrund der numerischen Chromosomenabweichungen prognostisch weiter zu unterscheiden.

\section{Th. M. de Reijke berichtete über}

\section{„Die transrektale Sonographie zur Früherkennung des Prostatakarzinoms"}

\section{Koautoren waren K. H. Kurth, N. F. Dabhoiwala, P. L. M. Vijverberg, E. A. J.M. de Jong}

Die transrektale Echographie wird heute vornehmlich zur Bestimmung der T-Kategorie des Prostatakarzinoms eingesetzt, um bei Fehlen von hämatogenen oder lymphogenen Metastasen das lokale Behandlungsverfahren sinnvoll zu planen.

Die Untersuchungsmethode der Prostata mit transrektaler Echographie war in den zurückliegenden drei Jahren Teil der Routineuntersuchung bei Patienten mit Erkrankung der Prostata. Sie erfolgte bei karzinomverdächtigem Palpationsbefund, chronischer Prostatitis und bei Patienten mit nach dem Palpationsbefund benignen Prostataadenom zur Volumenbestimmung, sowie bei Patienten mit erhöhten Tumormarkern (PSA, PAP) ohne suspekten Palpationsbefund. Die Screeninguntersuchung wurde im allgemeinen kombiniert mit ultraschallgesteuerter perinealer Prostatabiopsie bei echoarmem karzinomverdächtigen Befund, und/oder bei digital suspekter Prostata. Es wurde eine Echosonde mit einer Frequenz von $4 \mathrm{MHz}$ verwandt. 272 Patienten wurden untersucht, bei 190 Patienten fand sich ein echoarmes Gebiet, bei 82 Patienten wurde echographisch kein abweichender Befund erhoben. Sensitivität, Spezifität, positiver und negativer Voraussagewert wurden ermittelt und anschließend gegenübergestellt den gleichen Parametern bestimmt mit rektaler Palpation oder dem Tumormarker saure Prostataphosphatase (Tab.1). Hierbei zeigt sich, daß sowohl mit der rektalen Palpation als auch mit der Sonographie der Anteil falsch-negativer Befunde gering ist, ablesbar an einer Sensitivität von 94,1\% für die Sonographie und 92,9\% für die Palpation. Mit beiden Unter-

\begin{tabular}{l|l|l|c|l|l|l}
\hline & Sonographie & $\%$ & Palpation & $\%$ & PAP & $\%$ \\
\hline Sensitivität & $80 / 85$ & 94,1 & $79 / 85$ & 92,9 & $42 / 81$ & 51,9 \\
Spezifität & $77 / 187$ & 41,2 & $101 / 187$ & 54 & $105 / 170$ & 61,8 \\
pos. Voraussagewert & $80 / 190$ & 42,1 & $79 / 165$ & 47,8 & $42 / 107$ & 39,2 \\
neg. Voraussagewert & $77 / 82$ & 93,9 & $101 / 107$ & 94,4 & $105 / 144$ & 72,9 \\
\hline
\end{tabular}

Tab. 1 Diagnose des Prostatakarzinoms mit Sonographie $(n=272)$, digitaler Palpation $(n=272)$ oder PAP $(n=251)$ 
suchungen liegt der Anteil falsch-positiver Befunde relativ hoch. Die Spezifität ist bei der Sonographie geringer als bei der rektalen Untersuchung. Ein falsch-positiver Befund wurde häufiger echographisch erhoben. Die Spezifizität für die Sonographie betrug nur $42,1 \%$. Aus diesen Befunden, die sich weitgehend decken mit Mitteilungen in der Literatur, kann der Schluß gezogen werden, da $\beta$ die transrektale Echographie ungeeignet ist zur Screeninguntersuchung vornehmlich wegen der geringen Spezifität des Untersuchungsverfahrens. Eine zweite noch laufende Untersuchung, bei der die multiplan arbeitende 7-MHz-Sonde eingesetzt wurde, führte zu gleichen Ergebnissen.

Vorteile der transrektalen Echographie sind vor allem zu finden bei ihrer Verwendung zur echogesteuerten Biopsie oder zur Implantation von radioaktivem Material (J-125) zur interstitiellen Strahlentherapie und zur Festlegung der klinischen T-Kategorie. Eine relativ hohe Spezifität kann auch bei der Bestimmung der Tumorausbreitung erreicht werden (75\%).

\section{E. Allhoff, Urologische Universitätsklinik Hannover, behandelte}

\section{„Prognostische Faktoren des Hodentumors“}

Koautoren waren S. Liedke, C. Wittekind, P. Anton, H. Tanke und U. Jonas

Unter dem Gesichtspunkt der „Wait-and-see policy“ bei Hodentumoren im klinischen Stadium I, so wie es von Peckham vorgestellt wurde, kommt prognostischen Faktoren, die eine Diskriminierung von Patienten mit hohem Risiko auf Mikrometastasen gegenüber Patienten mit geringerem Risiko erlaubt, besondere Bedeutung zu. Die „wait-andsee“-Haltung bei Patienten im Stadium I des Hodentumors sieht vor, daß Patienten nach Behandlung des Primärtumors durch Kastration sich einer strikten Verlaufskontrolle unterziehen, um bei frühzeitigem Nachweis von Metastasen unmittelbar mit der Chemotherapie starten zu können. Bei früh entdeckten Metastasen dürfte es sich stets um „lowvolume"-Metastasen handeln, die in praktisch $100 \%$ erfolgreich mit Chemotherapie behandelt werden können. Im Stadium I kann bei ca. $25 \%$ aller Patienten erwartet werden, daß im späteren Verlauf Metastasen auftreten, in ungefährt $18 \%$ retroperitoneal, in einigen Fällen kombiniert mit Lungenmetastasen, in ca. $10 \%$ werden lediglich Lungenmetastasen gefunden. Würden alle diesen Patienten, so wie von den Gegnern der Wait-and-see policy verlangt, einer retroperitonealen Lymphknotendissektion unterworfen, würden bestenfalls $18 \%$ aller Patienten hiervon profitieren, jedoch nicht jene Patienten, die Lungenmetastasen entwikkeln, oder jene $75 \%$, die metastasenfrei bleiben. Bei 112 Patienten im Stadium I, die in Hannover in ein Wait-andsee-Protokoll aufgenommen wurden, wurde Tumorprogression (Metastasen) bei 38 Patienten (34\%) beobachtet. Die Häufigkeit lag somit höher als in anderen publizierten Serien. Zur Ermittlung des Risikos einer Tumorprogression wurde bei 50 Patienten (15 mit Tumorprogression, $35 \mathrm{~Pa}$ tienten ohne Progression) der Primärtumor auf seine Morphologie histologisch, Tumorgröße, T-Kategorie, Vaso-invasivität unter Verwendung eines endothelialen Markers untersucht und die prognostische Bedeutung jedes Einzelfaktors für die Progression bestimmt. Darüber hinaus wur- den Zellsuspensionen in einem automatisierten bildgebenden Analysesystem (MIAC. Leitz, Wetzlar) zur Bestimmung der Ploidie untersucht und im Verlauf die Tumormarker Alphafetoprotein und Beta-HCG bestimmt.

Lediglich der Nachweis von Hyperpentaploidie lie $\beta$ sich signifikant mit einem ungünstigen Verlauf korrelieren. Hyperpentaploidie fand sich bei $66,6 \%$ der $\mathrm{Pa}$ tienten mit Tumorprogression und lediglich bei $22,8 \%$ der Patienten ohne Tumorprogression. Keiner der übrigen prognostischen Faktoren ließ sich eindeutig dem klinischen Verlauf korrelieren. Die Autoren folgern daher, daß in ihren Händen lediglich die > 5c-Fraktion, bestimmt mit dem MIAC-System, eine prognostische Aussage über den weiteren Verlauf von Patienten mit Hodentumoren im Stadium I zuließ.

\section{Erektile Dysfunktion}

\section{K. P. Jünemann, Universitätsklinik Mannheim, berichtete} über

\section{„Pathophysiologie der erektilen Dysfunktion mit besonderer Berücksichtigung der neurologischen Leitungsbahnen und der glatten Schwellkörpermuskulatur“}

Koautoren dieser Untersuchung waren Persson-Jünemann und T. Lue

Seit Einführung der Pharmakotherapie mit vasoaktiven Substanzen zur Behandlung der erektilen Dysfunktion haben sich diagnostische und therapeutische Möglichkeiten dieses Krankheitsbildes aufgrund neuartiger Forschungsergebnisse völlig gewandelt. Über 44000 dokumentierte und publizierte Schwellkörperautoinjektionen sprechen für sich. Als unbefriedigend müssen jedoch die operativen Behandlungsformen, insbesondere die venenchirurgischen Operationstechniken, betrachtet werden, da im LangzeitFollow-Up die Erfolgsrate lediglich $39 \%$ beträgt. Es stellt sich somit die Frage nach der dominierenden Pathogenese der vaskulären Erektionsstörungen. Einen Einblick in die mögliche Pathologie und den daraus resultierenden Pathomechanismus brachte die von Persson-Jünemann und Lue durchgeführte elektronenmikroskopische Untersuchung bioptisch entnommenen Schwellkörpergewebes von $\mathrm{Pa}$ tienten mit vaskulärer erektiler Dysfunktion. Ziel dieser Studie war es, die penile Ultrastruktur der impotenten Männer in Korrelation zu setzen zu deren klinischen Untersuchungsergebnis, ermittelt mit Duplexsonographie und Pharmakontestung.

Bei 32 Patienten, die sich einer klinischen Diagnostik unterzogen, wurde im Rahmen einer Penisprothesenimplantation eine Schwellkörperbiopsie entnommen, die ohne Kenntnis der klinischen Daten elektronenmikroskopisch aufgearbeitet wurde. Anhand der ultrastrukturellen, morphologischen Veränderungen wurde eine Klassifizierung der einzelnen Patienten vorgenommen und nach Abschluß der Studie mit deren klinischen Ergebnissen verglichen. 
Die klinischen Befundergebnisse stimmten in allen Fällen mit den ultramorphologischen Befunden überein. Es konnten drei Gruppen unterschieden werden: 1. nicht-vaskuläre Erektionsstörung, 2. mäßiggradig ausgebildete arteriogene und 3 . hochgradig ausgebildete arteriogene erektile Dysfunktion. Gruppe 1 umfaßte das Klientel mit psychogen/neurogener Erektionsstörungen, die in allen drei untersuchten Fällen einen normalen Aufbau der glatten Schwellkörpermuskulatur zeigten. Erwartungsgemäß fanden sich pathologische Veränderungen an den intrakavernösen Nervenfasern. Die Gruppe 2 mit mäßiggradig ausgebildeter arteriogener Erektionsstörung zeigte hinsichtlich Anzahl und Verteilung der morphologischen Elemente einen Normalbefund. Ausgeprägte Veränderungen fanden sich allerdings intrazellulär im Bereich der zytoplasmatischen Strukturen der glatten Muskelzelle des Schwellkörpers. Neben einer ausgeprägten Mitochondrienvermehrung und -aggregation fand sich eine ausgedehnte Vakuolisierung in der Zellperipherie. Auffällig war zudem der intrazelluläre Verlust von Myofilamenten. Dieser Befund entsprach am ehesten einer veränderten Stoffwechselsituation, auf die die glatte Muskelzelle etwa durch Mitochondrienvermehrung reagiert. Patienten dieser Gruppe reagierten auf die intrakavernöse Papaverinapplikation mit einer maximalen Tumeszenz (E 2 bis E3). In der Gruppe der sogenannten SKAT-Nonresponder fanden sich ausgeprägte morphologische Veränderungen der glatten Schwellkörpermuskulatur sowohl intrazellulär als auch extrazellulär. Zum Teil fanden sich vollständige Zellmembrandestruktionen mit Zellkerndeformierungen bis hin zum nahezu vollständigen Verlust der kontraktilen Myofilamenten. Bindegewebe ersetzte aktives glattmuskuläres Schwellkörpergewebe. Auch im Bereich des Endothels der Sinusoidalräume wurden ähnliche Veränderungen festgestellt. Signifikante Verkalkungen oder Verschlüsse an den kleinsten Arteriolen fanden sich erstaunlicherweise nicht. Hieraus wurde gefolgert, daß die Ursache der vaskulären Erektionsstörung, insbesondere der arteriogenen erektilen Dysfunktion, nicht nur in den Arterien zu suchen ist, sondern vielmehr auf einer ausgeprägten Pathologie der glatten Schwellkörpermuskulatur beruht. Die massive intrakorporale Narbenbildung führt zu einem Relaxationsverlust der glatten Muskelzellen des erektilen Gewebes, dies wiederum führt zu einer kavernös/venösen Insuffizienz. Die Autoren stellen folgerichtig dann auch die Frage, inwieweit das sogenannte venöse Leck nicht Resultat einer Veränderung pathologischer Venen, sondern vielmehr einer relaxations-beeinträchtigten Schwellkörpermuskulatur ist.

Neben den vaskulär bedingten Erektionsstörungen bereitet insbesondere die Differentialdiagnostik der nicht-vaskulären Impotenz mitunter größere Probleme. Auch hier gilt, daß zum besseren Verständnis der möglichen diagnostischen Ansätze die Neurophysiologie und Neuropathophysiologie der reflexogenen Erektion unumgänglich sind. Aufgrund tierexperimenteller Studien konnte ein neurophysiologisches Konzept des Erektionsmechanismus erstellt werden. Mittels Elektrostimulation unterschiedlicher Nervenfasern bzw. Nervensysteme wurde deren Einfluß auf das erektile Gewebe bzw. Erektionsmechanismus untersucht. Die Stimulation der primär parasympathischen Nervi cavernosi, die dem sakralen Erektionszentrum S2 bis S4 entspringen, führt zu einer Peniselongation als Folge glattmuskulärer Schwellkörperrelaxation. Durch die gleichzeitige arterielle Dilatation nimmt der Bluteinstrom zu und führt aufgrund der Wiederstandsab- und Volumenzunahme zu einer venösen Restriktion. Die additive Elektrostimulation somatomotorischer Fasern des Nervus Pudendus resultiert in einer vollständigen Penisrigidität mit Schwellkörperdruckwerten, die weit über dem systemischen Blutdruck liegen. Schwellkörperdetumeszenz bzw. eine Erektionsunterdrückung wurde über die zusätzliche sympathische Nervus hypogastricus-Stimulation erreicht, die eine Schwellkörpermuskelkontraktion bzw. Relaxationshemmung zur Folge hatte. Dieser Mechanismus dürfte insbesondere bei der streßbedingten, psychogenen Erektionsstörung eine bedeutende Rolle spielen. Die reflexogene Erektion ist durch drei unterschiedliche Mechanismen zu erklären: 1. Der primär vaskuläre Mechanismus, der parasympathischen Einflüssen unterliegt und für Initiierung und Aufrechterhaltung der Erektion verantwortlich ist. 2. Die penile Rigidität, die einem somatomotorisch kontrollierten muskulären Mechanismus entspricht sowie 3. die Detumeszenz als vornehmlich sympathisch bedingtes Phänomen, welches als inhibitorischer Mechanismus bezeichnet werden kann. Die Kenntnis der neurophysiologischen Erektionsabläufe sollte dazu beitragen, ein suffizientes Diagnostikkonzept für die Evaluierung des autonomen Nervensystems der glatten Schwellkörpermuskulatur zu erreichen.

\section{S. C. Müller berichtete über}

\section{„Vergleich von Angiographie, penobrachialem Index und gepulster Dopplersonographie bei erektiler Dysfunktion“}

Koautoren waren Th. Fritz, J. Rüth, H. Schild und R. Hohenfellner, Urologische Universitätsklinik Mainz

Der erektilen Dysfunktion liegen meist organische Störungen zugrunde, unter diesen sind die vaskulären Erkrankungen am häufigsten beteiligt. In einer prospektiven Untersuchung wurden drei Untersuchungstechniken angewandt, um ihre Aussagekraft bei der Zustandsbeurteilung des penilen-arteriellen System zu ermitteln. Die Phalloarteriographie wurde bei 43 Patienten mit erektiler Dysfunktion vor und nach intrakavernöser Injektion von $15 \mathrm{mg}$ Papaverin und 0,5 mg Regitin durchgeführt. Bei 23 Patienten erfolgte die Bestimmung des penobrachialen Index (PBI), zusätzlich erfolgte die Duplexsonographie mit dem gepulsten Doppler.

Arteriographisch wurden bei $63 \%$ der Patienten Verschlüsse oder Stenosen der pudendalen oder penilen Arterien nachgewiesen. Eine Korrelation der Phalloarteriographie und des PBI zeigte sich jedoch nur in 39\%, während Arteriographie und Duplexsonographie in $90 \%$ übereinstimmten. Die Autoren schlossen aus diesen Ergebnissen, daß der PBI kein geeigneter Parameter zur Bestimmung des Zustandes der Corpora Cavernosa ist. Die Duplexsonographie ist in der Aussagekraft der Arteriographie gleichwertig, ist dieser jedoch vorzuziehen, da es sich um eine nicht-invasive Untersuchungsmethode handelt und hiermit in einem Untersuchungsgang Durchblutung, Morphologie der Corpora Cavernosa und Funktionszustand ermittelt werden können. Veränderungen des Gefäßdurchmessers und Blutstromgeschwindigkeit können mit der Duplexsonographie und der gepulsten Doppleranalyse objek- 
tiv nach Injektion von Papaverin und Regitin gemessen werden. In der Tumeszenzphase zeigt sich initial ein zweifacher Anstieg des inneren Durchmessers der Arteria Cavernosa mit konzentrischen Pulsationen und eine Blutstromgeschwindigkeit von minimal $25 \mathrm{~cm} / \mathrm{s}$. Bei erreichter Vollerektion werden die Arteria cavernosae komprimiert und ist nur noch ein minimaler Blutstrom festzustellen, vergleichbar dem erschlafften Zustand. Physiologische und pathologische Reaktionen auf die intrakavernöse Injektion von vasoaktiven Substanzen können zuverlässig mit der Dopplersonographie dokumentiert werden. Eine Unterscheidung zwischen angiogener und psychogener Impotenz ist somit möglich.

In einem zweiten Beitrag der gleichen Arbeitsgruppe wurden erste Ergebnisse der „perkutanen Penilen Okklusion" vorgestellt. Die unbefriedigenden Langzeitergebnisse der Venenligatur zur Behandlung der erektilen Impotenz bei venösem Leck stimulierte die Untersucher alternative Behandlungsmethoden anzuwenden. Seit 1988 wurden 17 Patienten mit erektiler Dysfunktion bei nachgewiesenem venösen Leck durch perkutane transluminale Venenokklusion behandelt. Hierbei wurden Coils und sklerosierende Substanzen verwandt. Der Zugang erfolgte über die tiefe dorsale Penisvenen oder (bei 4 Patienten) retrograd über die Vena Femoralis. Alle Patienten waren vorab mittels Duplexsonographie und Pharmakokavernographie/Kavernometrie abgeklärt.

Nach einem maximalen Followup von 23 Monaten betrug die Erfolgsrate bisher $65 \%$. Sechs Patienten hatten nach der Venenokklusion spontane Erektionen und waren kohabitationsfähig. Fünf Patienten erreichten Kohabitationsfähigkeit nach intrakavernöser Injektion vasoaktiver Substanzen. Fünf Patienten blieben impotent, ein Patient konnte nicht weiter verfolgt werden. Es wurden keine Komplikationen nach der Gefäßmanipulation beobachtet. Es bleibt abzuwarten, wie von den Autoren richtig unterstrichen, ob sich auf lange Sicht dieses Verfahren bewährt. Ebenso wie bei venösem Leck Rezidivoperationen möglich sind, kann auch die Venenokklusion wiederholt werden.

\section{$H$. Garibiyan stellte seine experimentellen Untersuchungen} über

\section{„Arterielle Gefäßanastomosen des Penis“}

vor. Die Untersuchungen erfolgten an der Urologischen Universitätsklinik Amsterdam und in San Fransisco. Koautoren waren T. F. Lue und E. A. Tanagho

Impotenzuntersuchungen und Behandlung der Impotenz erfordern Verständnis über die arterielle Blutversórgung des Penis. Die Anwesenheit von Anastomosen zwischen der Arteria dorsalis penis und der Arteria penis profunda wird im Schrifttum kontrovers behandelt.

Mittels mikrochirurgischer Technik, die eine 5-25fache Vergrößerung des Situs ermöglichte, wurde bei zehn Beckenpräparaten erwachsener Männer das arterielle Gefäßnetz präpariert und nach möglichen Anastomosen zwischen tiefen und dorsalen Penisarterien gesucht. Bei 7 von den 10 Präparaten fand sich eine Abzweigung der dorsalen Arterien, die die Tunica albuginea penetrierte und im Corpus cavernosum mit der Arteria penis profunda anastomosierte. Darüber hinaus fanden sich in allen Präparaten mehrere Verzweigungen der kavernösen Arterien die die Tunica albuginea penetrierten. Sie versorgen das Bindegewebe des Penis und das Corpus spongiosum und weisen selbst wiederum Anastomosen auf mit circumflexen Verzweigungen der dorsalen Penisarterien und/oder mit der dorsalen arteriellen Arkade der Penisglans.

Kenntnisse dieser arteriellen Blutversorgung könnten sich als nützlich erweisen bei revaskulisierenden Eingriffen.

\section{Wespes, E. stellte Ergebnisse vor über}

„Immunhistochemischer Nachweis von VIP- und NPYNerven im menschlichen Penis“"

Koautoren waren S. Schiffmann, C. C. Schulman und J. J. Vanderhaeghen, Urologische Klinik und Laboratorium zur Untersuchung von Neuropeptiden, Erasmus Hospital, Universität Brüssel

Immunreaktive, vasoaktive intestinale Polypeptide (IR-VIP) und Neuropeptide Y 1-36 (IR-NPY) wurden in den Nervenfasern arterieller und venöser Gefäßwände und in glatten Muskelzellen des menschlichen Penis beobachtet. Material wurde post mortem und intraoperativ entnommen und licht- und elektronenmikroskopisch unter Anwendung immunhistochemischer Methoden untersucht. Zahlreiche IRVIP- und IR-NPY-positive Nervenfasern fanden sich konzentriert in den inneren Anteilen der Adventitia arterieller und venöser Gefäße (Abb.2). Im Corpus cavernosum und spongiosum lagen IR-VIP- und IR-NPY-positive Nervenfasern zwischen den glatten Muskelfasern um die sinusoidalen Räume. Es überwogen die NPY-positiven Elemente. Unter Verwendung von Immungold-Techniken nach Fixation des Gewebes und Untersuchungen auf ultrastruktureller Ebene ließen sich IR-VIP- und IR-NPY-positive Areale lediglich in den Granula der Nervenenden nachweisen. Die rela-

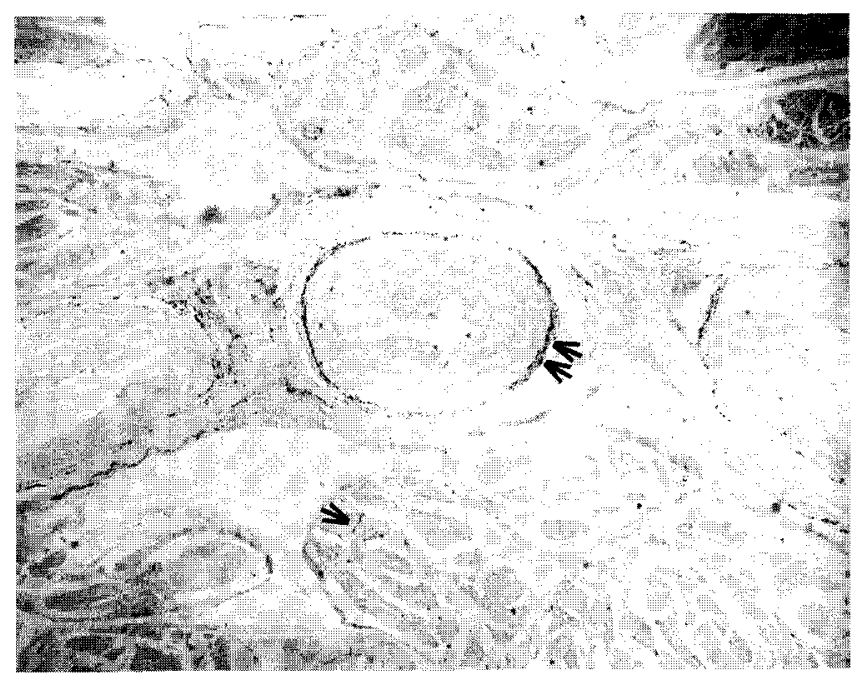

Abb. 2 ViP (intestinale polypeptide) und IR-NPY (Neuropeptide) immunhistochemisch dargestellt in der Adventitia von Gefäßen und zwischen den glatten Muskelfasern. 
xierende Wirkung von VIP auf die intrakavernösen glatten Muskelzellen wurde bereits demonstriert. NPY provoziert Kontraktionen der glatten Gefäßmuskelzelle in anderen Systemen und ist deshalb möglicherweise beteiligt bei der Detumeszenz und Erschlaffung des Penis. Die Verteilung der IR-VIP- und IR-NPY-Nervenfasern im Penis läßt vermuten, daß diesen Peptiden eine Aufgabe zukommt bei der Regulation des arteriellen und venösen Blutstroms, sowie des Tonus der glatten Muskelzelle intrakavernös während der penilen Erektion.

\section{Extrakorporeale Lithotripsie}

\section{P. L. M. Vijverberg berichtete über}

\section{„Erfahrungen mit einem mobilen Stoßwellentherapiegerät“"}

Koautoren waren Th. M. de Reijke, K. H. Kurth, H. J. Voogt

Seit Juni 1987 steht uns in den Niederlanden als erstem Zentrum ein mobiles Piezolith 2200-, später 2300-Gerät zur Verfügung. Neben der Möglichkeit des mobilen Einsatzes waren schmerzfreie Behandlung und die Steinortung durch Schallwellen statt durch Röntgen Gründe zur Anschaffung des Gerätes. In die Anschaffungskosten teilten sich vier Zentren. In der Erprobungsphase wurde das Gerät zunächst in den beiden Universitätskliniken von Amsterdam eingesetzt, später schlossen sich die Universitätsklinik Leiden und das Medizinische Zentrum Alkmaar an. Der Transport des Gerätes erfolgte mit einem hierfür speziell umgebautem Fahr- zeug. Die Aufstellung in den verschiedenen Behandlungszentren und die Überprüfung der Gerätefunktionen erfordern ungefähr 90 Minuten. Jedes Zentrum stellt einen eigenen Urologen, der für die Behandlung verantwortlich ist. Registration der Patienten, Anamnese und Behandlungsdaten werden zentral erfaßt und computerisiert verarbeitet. 1244 bisher behandelte Patienten wurden in diese Analyse aufgenommen. $74 \%$ aller Patienten wurden ambulant behandelt, lediglich Risikopatienten, Patienten mit Infektionen oder großen Ausgußsteinen oder Patienten, bei denen auxiliäre Maßnahmen erforderlich waren, wurden stationär therapiert. Gelegentlich erfolgte die EPL-Therapie unter Verarbeitung eines Spasmolitikums (1\%), allgemeine Narkose oder lokale Anästhesie waren nicht erforderlich. Nach einer sechsmonatigen Verlaufskontrolle waren $78 \%$ der Patienten steinfrei (oder zeigten Restfragmente $<2 \mathrm{~mm}$ ). Die Komplikationsrate bei ambulant behandelten Patienten war gering, lediglich $0,3 \%$ der Patienten wurden stationär aufgenommen wegen Fieber, bei 5,2\% wurde ein Pigtail plaziert. Die Häufigkeit der Mehrfachbehandlung des gleichen Steins betrug 28,5\%. Die mittlere Anzahl der verabreichten Schockwellen betrug 3658. Gerechnet über ein Jahr konnte wegen Reparaturmaßnahmen an insgesamt 5 geplanten Behandlungstagen das Gerät nicht eingesetzt werden. Aus unseren Ergebnissen folgern wir: 1. das verwandte Gerät eignet sich für den mobilen Einsatz, 2. bei adäquatem Transport ist kein nachteiliger Einfluß auf die Behandlungsresultate durch den mobilen Einsatz zu erwarten, 3. die narkose- und anästhesiefreie Behandlung ermöglicht bei $3 / 4$ aller Patienten poliklinische Therapie, 4. Überlegungen der Wirtschaftlichkeit und der Patientenakzeptanz stärken die Position eines mobilen Gerätes, das ohne anästhesiologische Maßnahmen eingesetzt werden kann. 